\title{
Action Coordination in Groups and Individuals: Learning Anticipatory Control
}

\author{
Günther Knoblich \\ Max Planck Institute for Psychological Research
}

\author{
Jerome Scott Jordan \\ Illinois State University
}

\begin{abstract}
When individuals act alone, they can internally coordinate the actions at hand. Such coordination is not feasible when individuals act together in a group. The present research examines to what extent groups encounter specific challenges when acting jointly and whether these challenges impede extending planning into the future. Individuals and groups carried out a tracking task that required learning a new anticipatory control strategy. The results show that groups face additional demands that are harder to overcome when planning needs to be extended into the future. Information about others' actions is a necessary condition for groups to effectively learn to extend their plans. Possible mechanisms for exerting and learning anticipatory control are discussed.
\end{abstract}

Researchers in the area of action planning and action control use a host of diverse tasks to investigate the cognitive functions that enable one to coordinate action alternatives. Examples include selecting and programming arbitrary actions in response to arbitrary stimuli (Hommel \& Prinz, 1997), switching between arbitrary tasks (Allport, 1993; Mayr \& Keele, 2000; Rogers \& Monsell, 1995), and carrying out two tasks at the same time (Meyer \& Kieras, 1997). Despite the differences between these tasks researchers tend to agree that action control involves a plan of some sort, be it an action effect (Hommel, Müsseler, Aschersleben, \& Prinz, 2001; Prinz, 1997), a task set (Allport, 1993), or a goal (Bekkering, Wohlschlaeger, \& Gattis, 2000). In addition, they tend to agree that the mechanisms underlying action coordination are internal to the actor.

Given this focus on internal mechanisms, it is interesting to note that groups of individuals can work toward a common goal without them (Steiner, 1972). Such a deficit is, of course, unimportant if planning and acting can be clearly separated and there are no coordination demands during the action phase. Under these conditions groups can overcome their lack of shared internal processes via the use of language (Clark, 1996). But if individuals have to time their actions in relation to those of another, language might be too slow, and the lack of shared internal processes may prove difficult to overcome. Under these conditions, group members might simply plan their actions in response to immediate error (i.e., current deviations from the goal state). While playing soccer for

Günther Knoblich, Cognition and Action, Max Planck Institute for Psychological Research, Munich, Germany; Jerome Scott Jordan, Deparment of Psychology, Illinois State University.

We thank Harold Bekkering, Rüdiger Flach, Chris Frith, Vittorio Gallese, Bernhard Hommel, Iring Koch, and Natalie Sebanz for helpful comments, and Irmgard Hagen, Patrick Back, and Lucia Kypcke for their help in collecting the data.

Correspondence concerning this article should be addressed to Günther Knoblich, Max-Planck-Institut für psychologische Forschung, Amalienstrasse 33, Munich 80799, Germany. E-mail: knoblich@psy.mpg.de example, teammates might coordinate passes by focusing on the consequences of the other members' actions and then react. Such a reactionary form of coordination will prove ineffective, however, if the timing demands are greater, as is the case in well-played soccer. Under these conditions effective coordination requires players to plan and execute their actions in relation to what they anticipate the other team members will do, not in response to what they have already done.

Given that groups are able to learn to engage in such anticipatory coordinations, despite their lack of shared internal processes, the purpose of the present article is to investigate the mechanisms underlying their ability to do so. We first examine what prior theories and research imply for joint action coordination. We then introduce a simple tracking task that allowed us to study individuals and groups under identical conditions and to derive several strategy and performance measures. The comparison of individuals and groups allowed us to determine the conditions under which groups can exert and acquire anticipatory coordination, despite the lack of shared internal processes.

\section{Prior Research}

In one study of group action coordination, R. C. Schmidt, Carello, and Turvey (1990) instructed 2 participants to swing their legs either in parallel (Persons 1 and 2 move one of their legs so that both legs arrive synchronously at the leftmost position, then at the rightmost position, etc.) or in symmetry (Persons 1 and 2 move one of their legs so that the legs meet in the middle, move outwards, etc.). These researchers found that at higher speeds groups fell into symmetry even when instructed to move in parallel. Similar results have regularly been reported for individuals in bimanual coordination tasks (Heuer, 1996; Kelso, 1997; Mechsner, Kerzel, Knoblich, \& Prinz, 2001). R. C. Schmidt et al. (1990) concluded from their results that the principles that govern coordination of multiple actions within individuals also hold for coordination across individuals.

Although R. C. Schmidt et al.'s (1990) account seems to hold for the tasks used in their experiment, we do not believe the task 
captures the unique difficulties groups must deal with when engaging in anticipatory coordination. To be sure, participants in the study needed to plan their actions in relation to the anticipated starting and stopping point of the partner's limbs. But the nature of the action to be produced was known. Thus, the only thing that had to be anticipated was the moment of a known action. In the anticipatory coordinations we proposed to investigate, group members have to plan their actions in relation to both the moment of the other's pending action as well as the type of action they anticipate the other will produce. For instance, well-played soccer requires that one pass the ball in anticipation of another player producing a particular action alternative (e.g., breaking left) at a particular time. Under these conditions one does not have access to the action alternatives of the other, and it is under these conditions that group coordination is most difficult.

A possible means of assessing how the external versus internal aspect of joint action might influence anticipatory coordination is to examine what different theories of individual action control have to say. One theory (R. A. Schmidt \& Lee, 1999) is based on the notion that individuals choose among action alternatives by waiting on action feedback from the environment and adjusting action selection and action timing accordingly. To be sure, this type of coordination is compensatory in nature (i.e., successive actions are selected on the basis of immediate feedback) and therefore may not seem to provide a means of modeling anticipatory coordination in groups. The classical literature on action control suggests, however, that compensation is the default in action control (R. A. Schmidt \& Lee, 1999), and research in the area of systems control indicates that (a) people prefer compensatory over anticipatory coordinations, and (b) it is very difficult to control dynamical systems in which certain actions have delayed effects on technical systems (Dörner, 1990; Reason, 1990). Thus, even though it is not theoretically clear how the use of a compensatory coordination might lead to the emergence of an anticipatory coordination, it may be the case that the former constitutes a necessary stage in the development of the latter. If this is the case, then learning an anticipatory coordination in a group might be much the same as learning one as an individual. That is, if immediate action feedback constitutes the essential factor underlying the learning of an anticipatory coordination and groups have just as much access to such feedback as does an individual, both should be able to learn an anticipatory coordination at roughly the same rate. In fact, groups might actually have an advantage because members of a group would be responsible for fewer action alternatives than would individuals.

Another approach to action coordination is the forward model approach, and it proposes that an individual coordinates action alternatives by predicting the sensory consequences of the alternatives and selecting among them on the basis of such predicted feedback (Blakemore \& Decety, 2001; Wolpert \& Ghahramani, 2000; Wolpert \& Kawato, 1998). Although this approach seems promising as an account of anticipatory group coordination, the extant literature is not conclusive as to how well even individuals are able to acquire such models. The postulated mechanisms are thought to bridge only the 200-ms delay that passes between action initiation and the arrival of the actual reafferent signal. It is not clear, therefore, how the temporal reference of a forward model could be extended beyond this interval to include anticipations regarding more temporally distal events. One possibility is sug- gested by Rosenbaum's (1980) research on action precuing. Specifically, he demonstrated that individuals are able to preprogram aspects of actions that are prespecified by external cues. This suggests that the temporal horizon of a forward model might be expandable if one is able to integrate anticipated and perceived events in such a way that action alternatives can be selected on the basis of anticipated as opposed to perceived events. For an individual, the states of all action alternatives would be internal. Thus, learning an anticipatory coordination would consist in appropriately relating currently perceived events, anticipated events, and action alternatives. For members of a group, who would not have access to all action alternatives, learning an anticipatory coordination would prove more difficult. They might be able to overcome this difficulty, however, if they had access to external cues that provided reliable information about the other members' action alternatives. Such cues could replace the internal information about action alternatives that are not at one's own disposal. In addition, this information could be (a) used to generate anticipations about what the other will do in a given situation and (b) taken into account when planning one's own actions. The following experimental paradigm was designed to address these issues.

\section{Experimental Paradigm}

We developed a task that allowed us to (a) assess individual and group performance under identical conditions and (b) create a conflict between compensatory and anticipatory coordination strategies (see Figure 1). This task consisted of keeping a circular ring stimulus (i.e., the tracker) on top of a smaller dot stimulus (i.e., the target). The target moved back and forth across a computer screen at a constant velocity and immediately reversed its course upon reaching either edge of the screen. The tracker was located in the same horizontal plane as the target.

Participants controlled its relationship to the target by pressing a right and a left key that incremented the tracker's velocity (to the right or left, respectively). Thus, if the tracker was moving to the right, a right keypress increased its velocity to the right, whereas a left keypress decreased its velocity to the right (i.e., increased its velocity to the left). All keypresses produced equal increments (decrements) in velocity. If, for instance, the tracker was moving to the right and a participant wanted to make it move to the left, he or she first had to press the left key repeatedly to reduce the tracker's rightward velocity to zero. Only if he or she continued to

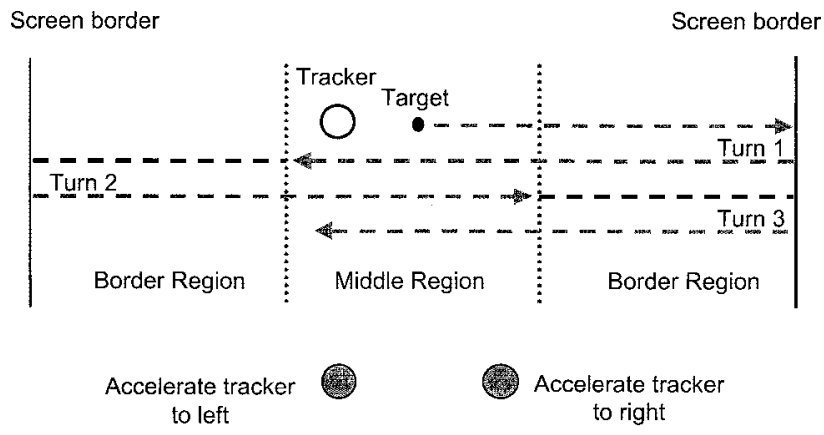

Figure 1. Illustration of experimental paradigm. 
press the left key would the tracker's velocity be incremented to the left.

Given that each keypress directly influenced the tracker's velocity, different regions of the tracking area required different patterns of keypresses. Within the middle region, a compensatory coordination strategy (CCS) was most effective. For instance, if the target was moving to the right and the tracker was left of the target, accelerating the tracker by a right keypress was the most effective action. In the border regions, a conflict arose between a CCS and an anticipatory coordination strategy (ACS). While using the CCS, one attempts to stay on target as long as possible. This strategy serves to minimize immediate error, and it does so until the target abruptly changes its direction of travel upon reaching the border. At this point, error begins to increase dramatically because the tracker can be decelerated only gradually. Several keypresses are then required to stop it and several more are needed to gain velocity in the opposite direction. During this interval, the target continues moving in the opposite direction, constantly increasing the distance between itself and the tracker. Thus, trying to minimize immediate error in the border regions creates a large future error. To overcome this error, one might use an ACS. Using this strategy, one decelerates the tracker before the target makes its turn. In this case, one is actually producing immediate error for the sake of minimizing future error. This is the case because the target continues to move toward the border as the tracker eventually comes to a halt. The key difference between the CCS and the ACS, therefore, is that in the former, selection among action alternatives is being determined by immediate feedback, whereas in the latter it is being determined by anticipated events.

\section{Research Questions and Predictions}

To determine (a) whether groups have additional difficulties with exerting and learning anticipatory coordination and (b) how they overcome these difficulties, we compared a condition in which one individual controlled both keys with a condition in which two individuals controlled one key each. Participants in the group condition wore headphones and were separated via a partition. This prevented them from using either spoken language or body language as a means of overcoming their lack of shared internal processes. This, in turn, allowed us to control each participant's access to external cues about the other's actions. We then manipulated the availability of such cues by providing half of the groups a tone that accompanied each keypress. This resulted in a $2 \times 2$ design in which individual and group conditions entailing such tones are referred to as the individual $(+)$ and the group $(+)$ conditions, whereas those not involving tones are referred to as the individual ( - ) and group (-) conditions. Each of the two keys was assigned its own unique tone. The tone therefore provided participants reliable information regarding the exact moment at which either of the two keys had been pressed. In the individual condition, the presence of the tones served to enhance the salience of the external feedback resulting from each keypress. In the group condition, however, the tones also had the potential of serving as a cue regarding the state of the other partner's action alternative (whether and when a key was pressed).

These manipulations afforded us the following hypotheses for general performance. If learning to affect an ACS is based more on salient immediate feedback than on the ability to generate appro- priate internal predictions, participants receiving more salient feedback (i.e., individual [+] and group [+] participants) should perform better than participants not receiving such feedback (i.e., individual $[-]$ and group $[-]$ participants). That is, they should give rise to less overall error in the border region. Or, if exerting and learning an ACS relies on internal predictions, participants in the individual conditions should perform better than those in the group conditions, at least initially. This is because participants in the individual conditions would have access to both action alternatives at all times, whereas those in the group conditions would have access to only one alternative. However, if participants in the group $(+)$ condition are able to use the tone as an external cue regarding the other participant's action alternative, they may be able to use such information to also generate predictions regarding the other's actions and ultimately perform better than group (-) participants.

Finally, to manipulate the need for an ACS, we manipulated the impact of the keypresses in such a way that on half the trials they produced a relatively large increment or decrement in tracker velocity. On the other half of the trials their impact was relatively lower. In addition, we crossed this manipulation with a two-level manipulation of target velocity. The crossing of these factors produced some trials in which the task was very difficult (i.e., high-impact keypresses coupled with fast target velocity) and others in which it was relatively simple (i.e., high-impact keypresses coupled with slow tracker velocity).

To be sure, general performance as expressed by tracker-target distance is only a rough measure of the extent to which participants successfully acquire and use an ACS. Several more specific measures of performance and strategy are available within the present task. Another performance-related index of the degree to which an ACS is successfully implemented is the position and the velocity of the tracker at the moment the target reaches the border and changes direction. The optimal strategy to minimize overall error is to bring the tracker to a stop (decelerate it to a velocity of zero) at a certain distance from the target in the moment in which the target turns. Therefore, deviations from the optimal position and zero velocity indicate how successfully an ACS was used.

The most direct strategy measure is the relative number of keypresses in the border region that lead to an increase in distance between tracker and target-what we refer to as the anticipatory brake rate. This constitutes a direct measure of the use of an ACS because it reveals the degree to which the participants' keypresses actually increase immediate error, as opposed to decreasing it. Because there is no reason to increase immediate error other than to avoid future error, the emergence and maintenance of anticipatory braking clearly indicates that participants are selecting successive actions on the basis of anticipated versus actual error.

Stopping the tracker requires that anticipatory brakes be produced in succession. This means that the nonbraking action alternative (i.e., the keypress that would decrease immediate error yet increase long-term error) has to be withheld. We hypothesized the following: For a participant in the individual condition who is learning or has already learned the ACS (i.e., he or she engages in anticipatory braking) withholding the nonbraking action alternative would not be difficult, for he or she has internal access to both alternatives at all times. For participants in the group conditions however, the situation is different. The action alternatives suggested by the ACS (i.e., anticipatory braking) and the CCS (i.e., 
offsetting immediate error) are not available in the same agent. They are distributed across two agents. Thus, the group member responsible for minimizing immediate error (CCS) might continue to do so as the member responsible for minimizing future error (ACS) attempts to brake. The conflict between these two alternatives can be directly measured by examining the degree to which anticipatory brakes are followed by interfering compensatory presses. We refer to this measure as the interference rate.

A further measure of the extent to which an ACS has been acquired and implemented is the time lag between consecutive anticipatory braking presses. We hypothesized the following: If the group member responsible for implementing the ACS (i.e., producing anticipatory braking keypresses) anticipates interference from the other member, he or she may produce successive braking keypresses as quickly as possible to overcome the partner's interference. If the braking member anticipates cooperation, however (i.e., he or she anticipates the other member will not produce interfering compensatory presses), he or she will not have to produce successive anticipatory braking presses as quickly as possible. Thus, the degree to which partners learn to produce anticipatory braking presses in anticipation of cooperation from their partner can be directly measured via the time lag between anticipatory brakes. Of course, participants in the individual condition have internal access to both action alternatives and thus should be able to produce anticipatory braking keypresses in anticipation of no interference. If, however, learning the ACS and how to implement it is a gradual process, individuals may actually oscillate between the CCS and the ACS as they attempt to learn the best moment at which to start producing anticipatory brakes. If this is the case, then one should see decreases in the number of interfering keypresses and increases in the time lag between anticipatory brakes as individuals come to acquire a more effective ACS.

\section{Method}

\section{Participants}

A total of 111 participants (41 male; 70 female), recruited by advertising at the University of Munich, Munich, Germany, and in local papers, took part in the study. Participants ranged in age from 19 to 35 years. All had normal or corrected-to-normal vision and received payment for their participation. They were randomly assigned to the four experimental conditions. Fifteen were assigned to the individual $(+)$ condition and 18 to the individual ( -$)$ condition. Thirty were assigned to the group (+) condition (forming 15 pairs) and 48 to the group $(-$ ) condition (forming 24 pairs). We collected more data for the group without tone condition because pilot experiments had shown that the variability was higher in this condition.

\section{Material and Procedure}

Upon entering the lab, participants were informed about the task. They were instructed individually in both the group and individual conditions. Afterward, they were seated in front of a computer monitor at a distance of $80 \mathrm{~cm}$. They wore a set of headphones. Participants in the group condition were divided by a partition. They could neither see nor talk to one another. However, each participant was provided with a separate computer monitor, and all events taking place during the experiment (e.g., the movements of the tracker and the movements of the target) were presented simultaneously on both monitors. Thus, the only information shared in both group conditions was the task display. All participants underwent 12 training trials.

Participants in the individual condition were given a control panel consisting of a left and a right key. They pushed them with their left and right hand, respectively. Left keypresses resulted in tracker acceleration to the left, and right keypresses resulted in tracker acceleration to the right. In the group condition, each member was given an individual control panel consisting of one key. Group members pushed the key with their dominant hand. Keypresses of the individual on the left side of the partition resulted in tracker acceleration to the left, whereas those of the other individual produced tracker acceleration to the right. In the individual $(+)$ and group $(+)$ conditions each right keypress triggered a $600-\mathrm{Hz}$ tone, and each left keypress triggered a $200-\mathrm{Hz}$ tone of $100-\mathrm{ms}$ duration. Tones were presented over the headphones.

The course of each trial was as follows. At the beginning, a solid, circular target (size $0.3^{\circ}$ of visual angle) and a transparent, circular tracker (size $1.0^{\circ}$ of visual angle) were displayed in the middle of the screen for $500 \mathrm{~ms}$, the tracker being superimposed on the target. Then, the target started moving with constant velocity. Initial target direction was counterbalanced. After reaching the border, the target abruptly traveled back in the opposite direction. There were three such target turns during each trial After the third turn, the target moved back to the middle of the screen and vanished. Initial tracker velocity was zero.

The experiment consisted of three blocks of 40 trials each. Target velocity and impact of keypress varied across the trials within each block. Target velocity was either slow $\left(3.3^{\circ}\right.$ per second $)$ or fast $\left(4.3^{\circ}\right.$ per second $)$, and the impact of keypress was either low (velocity change $0.7^{\circ}$ per second squared) or high $\left(1.0^{\circ}\right.$ per second squared). The order of trials was randomized within each block.

\section{Data Analysis}

We restricted the evaluation of all dependent variables to the border regions (the left or right third of the screen depending on the direction in which the target traveled) because the action conflict of interest arises only in these regions. Tracking performance before the first turn was not included in the analysis because in this phase of each trial the task requirements were different (e.g., accelerating the tracker from zero velocity). By restricting the analysis to Turns 2 and 3 we also ensured that each member in the group condition was responsible for braking at one turn and responsible for accelerating at the other.

We derived six dependent variables from the data, three to assess performance and three to assess acquisition and implementation of coordination strategies. As a measure of general performance, we computed the absolute distance between tracker and target in the border regions before the second and the third turn. Unlike most tracking paradigms, ours did not use the mean root square error (RSE) as the performance measure. The reason is that the RSE gives a larger weight to higher deviations, and thus if control over the tracker is lost, the error becomes enormous. As a consequence, the RSE would be largely biased by trials in which control over the tracker was lost. Therefore, the use of the absolute error is more appropriate. In addition, we analyzed tracker-target distance and tracker velocity at the moment the target reached the border and changed its direction of travel. Optimal performance was computed under the assumption of a maximal keypress rate of 5 keypresses per second separately for each combination of target velocity and impact of keypress.

To assess the extent to which an ACS was used, we derived three further dependent variables. The first was the percentage of anticipatory brakes in the border region. Our definition of an anticipatory keypress was as follows: (a) It had to occur while the tracker was on or behind the target, (b) it reduced tracker velocity, and (c) it did not occur right after a keypress that increased tracker velocity. The rationale for the third point (c) is that switches between two keys can also reflect a CCS that is directed toward keeping the tracker on target as close as possible. Hence, we used a 
conservative measure to avoid overestimating the number of keypresses reflecting an ACS. In the second step, we computed the rate of anticipatory brakes that were followed by an accelerating keypress relative to the sum of anticipatory keypresses in each trial. This variable we refer to as interference rate, because it provides a direct measure of the extent to which coordination problems were encountered. The third variable was the temporal distance between consecutive anticipatory brakes.

\section{Results}

Initial analyses revealed that there were no differences between the individual $(+)$ and $(-)$ conditions in any of the dependent variables. This was true for each single level of velocity and impact and throughout consecutive blocks. None of the differences approached significance (all $p \mathrm{~s}>.10$ ). This means that the additional auditory feedback did not systematically influence individuals' performance or strategy. Therefore, to simplify the analyses, we collapsed the data across these two conditions and compared individuals, groups with tones (group $[+]$ ), and groups without tones (group [-]).

Regarding task difficulty, there were only small differences between the low velocity-high impact, low velocity-low impact, and high velocity-high impact conditions. All three turned out to be relatively easy and qualitatively similar compared with the most difficult high velocity-low impact condition. Therefore, to further simplify the analyses, we collapsed the data across these three conditions. Thus, we analyzed two levels of task difficulty: difficult (high velocity-low impact) and easy (the remaining three conditions). Accordingly, each dependent variable was entered in a mixed 3 (condition: individual, group [+], group [-]) $\times 3$ (block: first, second, third) $\times 2$ (task difficulty: easy, difficult) analysis of variance (ANOVA). Condition was a between-subjects factor, and block and task difficulty were within-subject factors.

\section{General Performance}

Figure 2 shows the results for general performance. Panel A is for the easy condition and Panel B is for the difficult condition. Different points on the $x$-axis refer to different blocks. Accord- ingly, the slope of each line represents learning effects. The different lines represent the different experimental conditions. When the task was easy, substantial performance differences between the experimental conditions were present only during the first block. Performance was worst in the group (-) condition, intermediate in the group $(+)$ condition, and best in the individual condition. Later, participants in both group conditions approached the performance of individuals who performed well from the start. When the task was difficult, performance in both group conditions was initially equally worse than in the individual condition. However, during the second and third blocks, performance in the group $(+)$ condition approached that of the individual condition, whereas performance in the group (-) condition remained worse. In other words, groups receiving the tone feedback learned to perform as well as individuals, whereas groups sharing only the display did not.

The ANOVA revealed significant main effects for condition, $F(2,69)=10.1, p<.001$; block, $F(2,138)=83.9, p<.001$; and task difficulty, $F(2,138)=154.0, p<.001$. There was a significant Condition $\times$ Block interaction, $F(4,138)=3.9, p<.01$; a significant Condition $\times$ Task Difficulty interaction, $F(2$, $69)=5.8, p<.01$; and a significant Block $\times$ Task Difficulty interaction, $F(2,138)=43.1, p<.001$. The three-way interaction was not significant. We conducted Newman-Keuls tests to further assess the statistical significance of the differences observed in the difficult task condition. They showed that during the first block there was a significant performance difference between the individual and group $(+)$ conditions $(p<.05)$ and the individual and group $(-)$ conditions $(p<.05)$. However, there was no significant difference between the group conditions $(p=.64)$. During the last block, there was a significant difference between the group (-) and the individual conditions $(p<.01)$ and the individual and group $(+)$ conditions $(p<.01)$ but no significant difference between the individual and the group $(+)$ conditions $(p=.38)$.

Specific aspects of performance at target turn. Optimal performance dictates that the tracker should be stopped at a certain distance from the border at the moment the target turns. The next
A

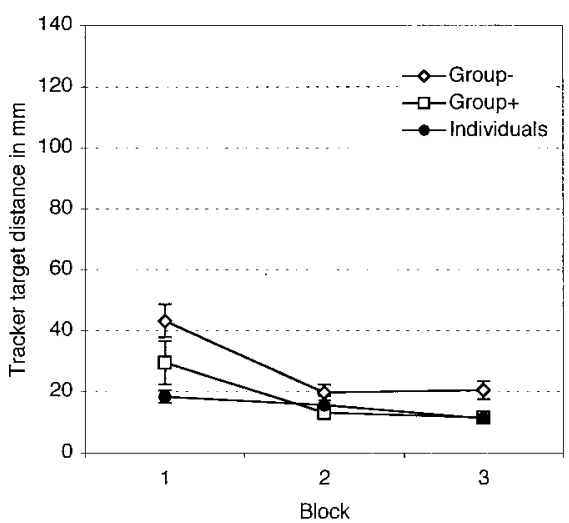

B

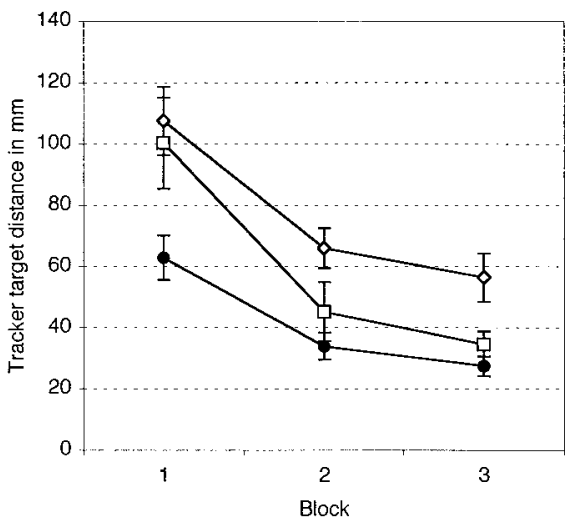

Figure 2. General performance: Tracker-target distance in border region across consecutive blocks in easy (A) and difficult (B) task conditions. In the group + condition a tone accompanied each keypress; in the groupcondition a tone did not accompany each keypress. 
two analyses assess how the participants in the different conditions differed in achieving the correct distance and the correct velocity.

Tracker-target distance. Figure 3 displays the result of our analysis of the tracker-target distance. When the task was easy (Figure 3A) the correct tracker-target distance could generally be achieved from the start. Only the participants in the group (-) condition had some problems in early trials. When the task was difficult (Figure 3B) participants in all conditions improved across consecutive blocks. During the initial block, the distance was less optimal in the group conditions than in the individual condition. In Blocks 2 and 3 there were no significant differences between experimental conditions. This implies that groups improved more across consecutive blocks.

The ANOVA showed significant main effects of block, $F(2$, $138)=33.6, p<.001$, and task difficulty, $F(1,69)=103.1, p<$ .001 . The main effect of condition failed to reach significance, $F(2$, $69)=2.9, p=.06$. There was also a significant Condition $\times$ Difficulty interaction, $F(1,69)=3.9, p<.05$, and a significant Condition $\times$ Block interaction, $F(4,138)=5.4, p<.001$. The three-way interaction was not significant. We conducted Newman-Keuls tests to further assess the statistical significance of the differences observed in the difficult task condition. During the initial block the tracker-target distance was significantly higher in groups than in individuals $(p<.05)$. During the last block, there were no significant differences between conditions (all $p \mathrm{~s}>.10$ ).

Tracker velocity. In the next step, we analyzed tracker velocity at target turn. Optimal performance dictates zero velocity at this point. Figure 4 illustrates the results.

The results demonstrate that controlling tracker velocity generally posed more of a challenge for groups than for individuals and that all participants improved across consecutive blocks. When the task was easy, participants in the individual condition were able to fully stop the tracker whereas participants in both group conditions were not. Similarly, when the task was difficult, participants in both group conditions continued to have more problems stopping the tracker all the way through the end of the experiment. However, whereas participants in the group (-) and individual conditions improved by a comparable amount, participants in the group
$(+)$ condition improved more. In short, they were better at stopping the tracker in the right moment than were participants in the group (-) condition. Nevertheless, they performed somewhat worse than individuals. Note that overall, individuals also did not manage to reduce tracker velocity to zero in the difficult condition.

The results of the ANOVA were as follows. There were significant main effects of condition, $F(2,69)=17.1, p<.001$; block, $F(2,138)=153.9, p<.001 ;$ and task difficulty, $F(1,69)=476.5$, $p<.001$. There was also a significant Condition $\times$ Block interaction, $F(4,138)=4.4, p<.01$; a significant Condition $\times$ Task Difficulty interaction, $F(2,69)=5.6, p<.01$; and a significant Block $\times$ Task Difficulty interaction, $F(2,138)=25.8, p<.001$. The three-way interaction was also significant, $F(4,138)=2.5$, $p<.05$. Again, we conducted Newman-Keuls tests to further assess the statistical significance of the differences observed in the difficult task condition. During the initial block, velocity was faster in both group conditions than in the individual condition (both $p s<.001$ ), and there was no significant difference between the two group conditions. During the last block velocity in the group $(+)$ condition was significantly lower than in the group $(-)$ condition $(p<.05)$ but still significantly higher than in the individual condition $(p<.01)$.

\section{Assessment of Control Strategies}

The preceding analyses addressed differences in performance. The remaining analyses address strategy measures that allowed us to directly assess to what extent an ACS was used and acquired in the different conditions.

Anticipatory brakes. Figure 5 shows the results of the analysis of the anticipatory brake rate. When the task was easy the anticipatory brake rate was highest in the individual condition, lower in the group (+) condition, and lowest in the group (-) condition. This pattern did not change across blocks. The results were different when the task was difficult. Initially, the anticipatory brake rate in both group conditions was much lower than that in the individual condition. The anticipatory brake rate increased by the same amount in the individual and group $(-)$ conditions across
A

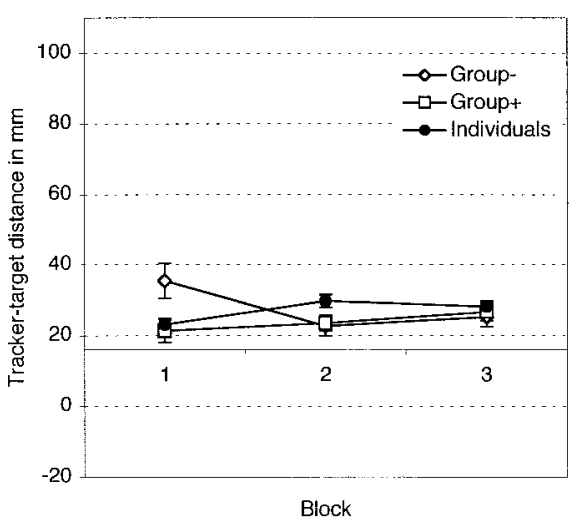

B

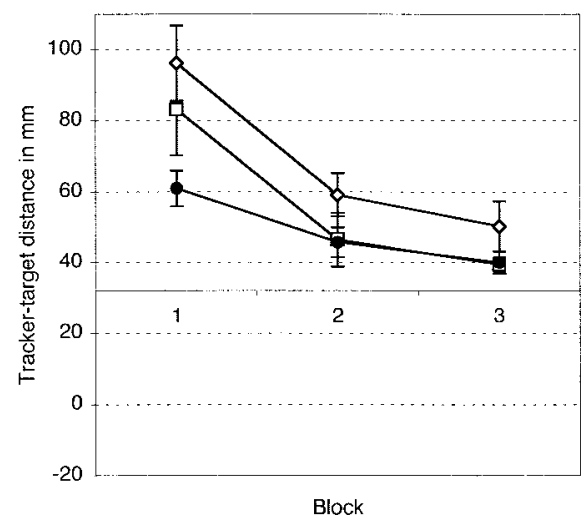

Figure 3. Tracker-target distance at target turn across consecutive blocks in easy (A) and difficult (B) task conditions. In the group + condition a tone accompanied each keypress; in the group - condition a tone did not accompany each keypress. 
A

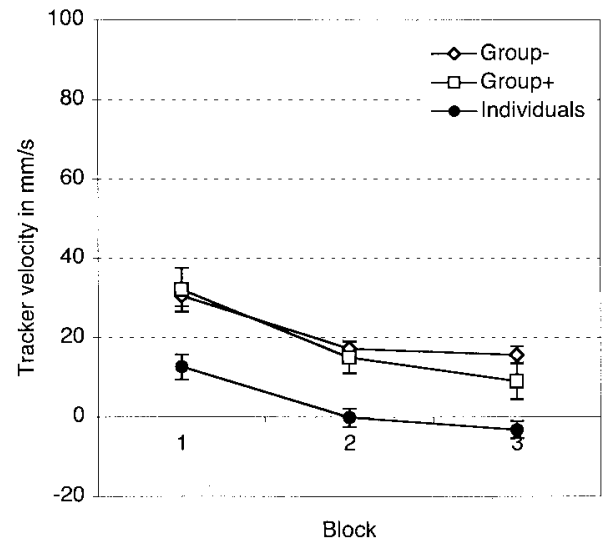

B

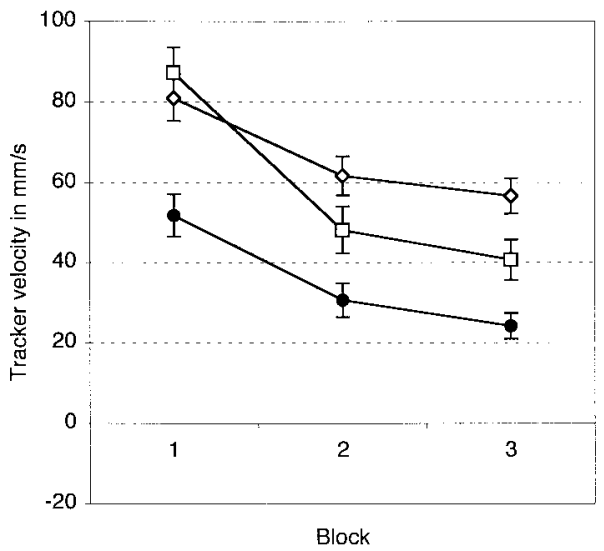

Figure 4. Tracker velocity at target turn across consecutive blocks in easy (A) and difficult (B) task conditions. In the group + condition a tone accompanied each keypress; in the group - condition a tone did not accompany each keypress.

consecutive blocks. However, the increase in anticipatory brakes was much larger in the group $(+)$ condition. During the last block, it became almost as high as that in the individual condition. Thus, groups receiving the auditory feedback learned to use an ACS to the same extent as did participants in the individual condition.

The ANOVA revealed significant main effects for condition, $F(2,69)=21.1, p<.001$; block, $F(2,138)=90.5, p<.001$; and task difficulty, $F(1,69)=5.9, p<.05$. In addition, there was a significant Condition $\times$ Block interaction, $F(4,138)=3.4, p<$ .05 ; a significant Condition $\times$ Difficulty interaction, $F(2$, $69)=4.2, p<.05$; and a significant Block $\times$ Difficulty interaction, $F(2,138)=66.2, p<.001$. The three-way interaction was also significant, $F(4,138)=4.4, p<.01$. Newman-Keuls tests were conducted to more closely assess the differences between experimental conditions in the difficult task condition. During the first block there was a significant difference between both group conditions and the individual condition (both $p \mathrm{~s}<.01$ ). During the last block, the group (+) anticipatory brake rate was significantly higher than the group $(-)$ rate $(p<.001)$ but not significantly lower than the individual brake rate $(p=.13)$.

Interference rate. This variable indicates how often a compensatory keypress interrupted a sequence of anticipatory brakes and therefore provides a direct measure of the extent to which coordination problems were encountered. Figure 6 illustrates the results.

When the task was easy the interference rate was lowest in the individual condition, higher in the group $(+)$ condition, and highest in the group (-) condition. When the task was difficult the interference rate was comparable in the group $(+)$ and individual conditions but much higher in the group $(-)$ condition. Regardless of task difficulty and experimental condition the interference rate slightly decreased across consecutive blocks.
A

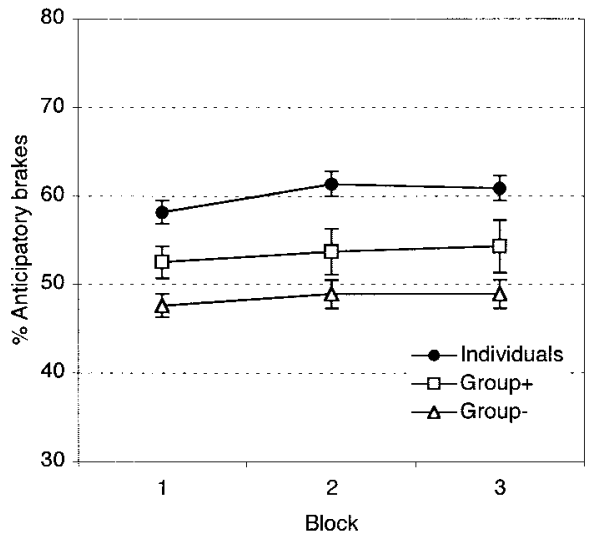

B

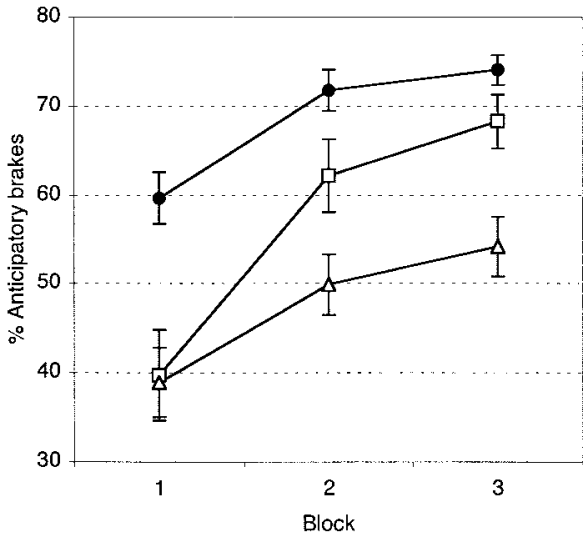

Figure 5. Anticipatory brake rate across consecutive blocks in easy (A) and difficult (B) task conditions. In the group + condition a tone accompanied each keypress; in the group - condition a tone did not accompany each keypress. 
A

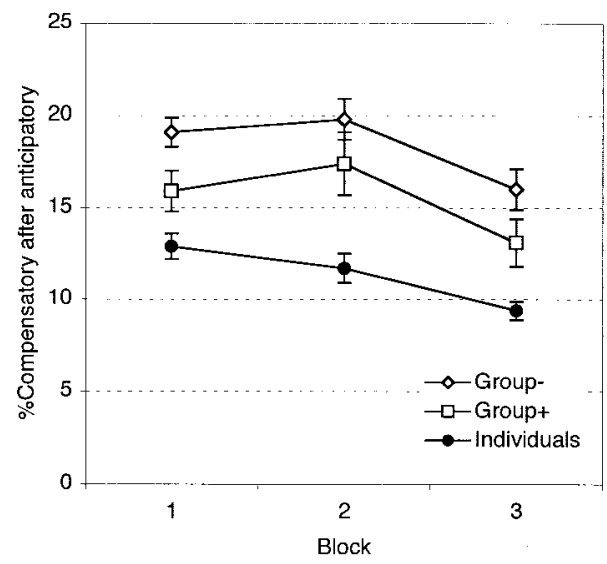

B

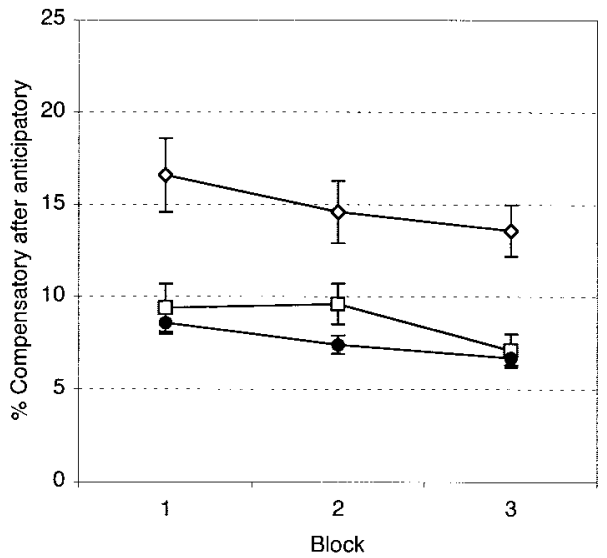

Figure 6. Percentage of anticipatory brakes followed by a compensatory keypress (interference rate) across consecutive blocks in easy (A) and difficult (B) experimental conditions. In the group + condition a tone accompanied each keypress; in the group - condition a tone did not accompany each keypress.

The ANOVA revealed significant main effects for condition, $F(2,69)=27.5, p<.001$; block, $F(2,138)=22.7, p<.001$; and task difficulty, $F(1,69)=68.1, p<.001$. There were no significant two-way or three-way interactions. The two-way Condition $\times$ Difficulty interaction fell short of reaching significance ( $p=.06$ ) despite the fact that the results for the three experimental conditions look quite different for the easy and difficult tasks. Therefore we conducted further Newman-Keuls tests to assess these differences. When the task was difficult the interference rate was higher in the group (-) condition than in the group (+) and the individual conditions (both $p \mathrm{~s}<.001$ ). There was no significant difference between the group $(+)$ and individual conditions ( $p=.43$ ). When the task was easy, the interference rate in the group $(+)$ condition was significantly higher than in the individual condition $(p<.001)$ and significantly lower than in the group $(-)$ condition $(p<.05)$.

\section{A}

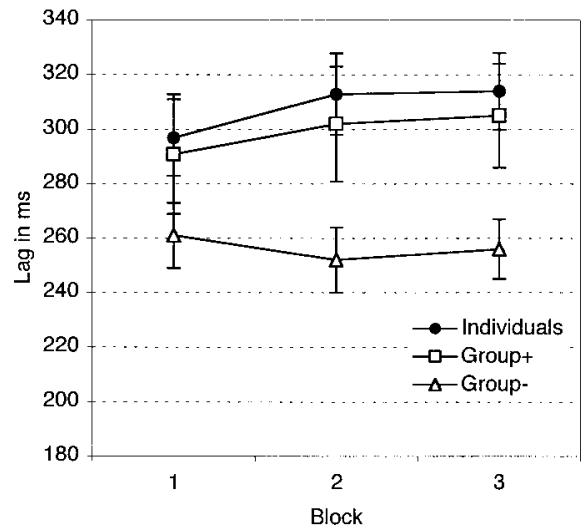

Lags between anticipatory brakes. As a last measure of the use of an ACS we analyzed the mean temporal interval between anticipatory brakes. Figure 7 illustrates the results of this analysis.

Lags were generally much shorter in the easy than in the difficult condition. When the task was easy, lags were considerably shorter in the group $(-)$ than in the group $(+)$ and individual conditions. This indicates that groups receiving no tone feedback experienced more time pressure, even when the task was easy. The lags remained relatively constant across consecutive blocks. The pattern was different when the task was difficult. During the initial trials, lags were short in all experimental conditions and there were no significant differences between conditions. Later, the lags became somewhat longer but only in the group (+) and individual conditions. Participants braking in the group $(-)$ condition pushed the keys as fast as they could until the end of the experiment.
B

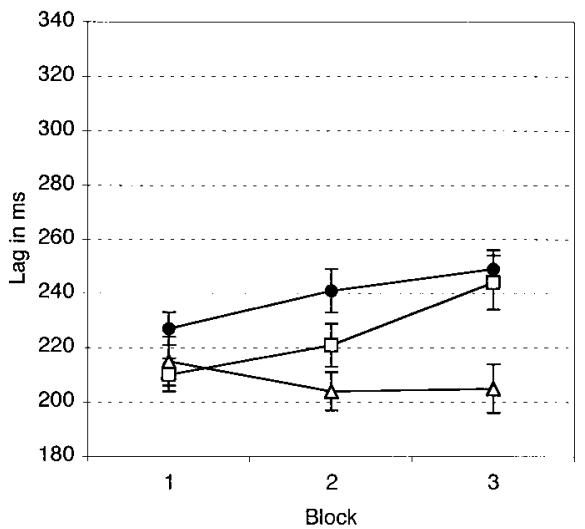

Figure 7. Lags between anticipatory keypresses across consecutive blocks in easy (A) and difficult (B) task conditions. In the group + condition a tone accompanied each keypress; in the group - condition a tone did not accompany each keypress. 
The ANOVA revealed significant main effects for condition, $F(2,69)=5.4, p<.01$; and task difficulty $, F(1,69)=83.8, p<$ .001 . The main effect for block failed to reach significance, $F(2$, $138)=3.0, p=.054$. Only the Block $\times$ Condition interaction was significant, $F(4,138)=3.1, p<.05$. Newman-Keuls tests confirmed that when the task was difficult the lags were significantly higher in the group (+) and individual conditions than in the group $(-)$ condition during the last block (both $p \mathrm{~s}<.01$ ).

\section{Discussion}

The results provide evidence for the following three claims. First, the saliency of action feedback did not affect the ability to use and learn an ACS. Second, groups encountered more coordination problems than did individuals; most of these problems derived from attempting to exert an ACS in the midst of increasing task demands. And third, groups were able to overcome their problems with learning and implementing an ACS if they were provided an external cue regarding the state of the partner's action alternative.

The first claim is supported by the lack of differences between participants receiving tones (individual $[+]$ and group $[+]$ ) and participants not receiving tones (individual [-] and Group [-] condition) in a variety of performance and strategy measures. If the saliency of action feedback had been the decisive factor for using and learning an ACS, one would expect the main differences to occur not between groups and individuals but between participants receiving and not receiving salient action feedback. The result is in accordance with the widely acknowledged fact that individuals use internal predictions to control the selection and timing of their actions (cf. Wolpert \& Ghahramani, 2000) whereas groups with distributed action alternatives can obviously not directly derive predictions for action alternatives not at their disposal.

The second claim, that groups encounter more coordination problems than do individuals, is also supported by several results. Initial group performance was generally poorer than individual performance, and groups did not benefit from the external cues (tones). The closer analysis of two specific aspects of performance, tracker position and tracker velocity at target turn, revealed that groups found it easier to optimize tracker position than tracker velocity. These results illustrate that the challenge for groups consisted specifically in the control of the dynamic aspects of the tracker movement. The differences in the easy task condition were small compared with the huge differences in the difficult task condition, in which anticipatory control was required to a large extent. Accordingly, initially groups had specific problems to control tracker velocity in the difficult task condition. Furthermore, the initial differences in the anticipatory brake rates between groups and individuals were immense.

The third claim, that external cues about the other group member's actions allowed groups to effectively implement an ACS, is also supported by several results. Although the availability of such information did not affect performance initially, it had a large impact on performance during later trials. In fact, groups receiving external cues became more similar to individuals than did groups that did not. First, their overall performance became almost indistinguishable from that of individuals. Second, they produced almost the same anticipatory brake rate as did individuals in later trials, although they had started out as low as groups receiving no external cues during the initial trials. Third, the analysis of the interference rate illustrated that keypresses interfering with anticipatory braking could be avoided. All of these results demonstrate that groups receiving external cues learned to implement the ACS to almost the same extent as did individuals. The only aspect of the task that groups seemed to find more challenging was stopping the tracker at the moment of target turn.

This pattern of results cannot be explained by the assumption that external cues about the other's actions simply made the task easier. The interference rate for groups receiving external cues was as low as the one for individuals from the start. Nevertheless, initial performance was as poor as in groups receiving no external cues. If external cues had simply made the task easier, participants in the group $(+)$ condition should have been comparable to individuals from the start. The observed pattern of results can be explained only by the assumption that an ACS was acquired.

The pattern of results for participants in the group (-) condition remained quite different from all other conditions until the end of the experiment. Participants in the group (-) condition could increase their anticipatory brake rate only to a small extent, because they kept interfering with the other during the braking phase. Because interference could not be avoided, group members in this condition worked hard to optimize the only parameter fully under their individual control, that is, the velocity of subsequent keypresses required for stopping the target. The lag between consecutive brakes was much shorter than for the other experimental conditions. It is possible that applying this individual-centered strategy actually kept performance lower, because participants were so busy trying to achieve high keypress rates that they did not attend to other aspects of the task. Alternatively, high keypress rates might have been the only way to indicate to the other to not act. In short, participants in this condition worked harder and achieved less, because they could implement the ACS to a lesser extent.

A further important aspect of the results is that in the difficult task condition the interference rate was generally lower for groups receiving external cues as compared with groups who did not. At the same time, the anticipatory brake rates were the same in both group conditions, during the initial trials. Later, only the group with additional information achieved the high rates necessary for successful performance. This means that the external cues were immediately used to avoid action conflicts. But using the external cues as a stop signal was not sufficient to more successfully implement the ACS. Group members who had the signals started braking too late and continued to accelerate too long. Hence, it is likely that the external cues also acted as a means to generate anticipations about the other's actions.

To summarize, the results of the present study seem to support the assumption that anticipations about the other's actions underlie the ability to jointly learn and implement an ACS. If immediate feedback had been the critical factor, participants receiving tones (i.e., individual [+] and group [+] participants) should have been better able to exert anticipatory control than those not receiving tones. The fact that the tones did not influence strategy and performance of those in the individual condition yet served to enhance the performance of those in the group $(+)$ condition indicates that the external cue allowed group $(+)$ participants to extend the temporal horizon of planning, presumably by generating anticipations about the other person's actions. Participants in 
the individual condition did not need such a cue, for they had internal access to the state of all action alternatives at all times and were thus able to relate perceived events, action alternatives, and anticipated events from the start.

\section{Possible Mechanisms for Learning Anticipatory Control}

These results raise two important theoretical issues. First, what are the mechanisms by which anticipated events come to be more important than current events in action control, especially when the current events suggest an action alternative that is different from the one required to achieve successful overall performance? Second, how can information about another's actions become integrated with information about one's own? In the following, we address these questions in turn.

One possible means of accounting for the shift from selecting actions on the basis of immediate events to selecting them on the basis of more temporally distal consequences is to assume that predictions are generated for all action alternatives that can be applied to a certain task. A selection mechanism would then be responsible for selecting the action whose predicted consequences most closely match the goal. The most prominent general theory postulating such processes is the forward model account (Wolpert \& Kawato, 1998). Extending the temporal horizon of action planning, according to this account, would consist of an extension of the temporal distality of the prediction for each of the action alternatives.

Another possibility is that the extension of the temporal horizon derives from a temporal expansion of the goal itself. This would imply that there is an additional mechanism that produces such expansion by integrating information across larger time scales to enable the prediction of critical intended events (e.g., the position and velocity of the tracker at target turn in the present task). Hence, the predictions for each single action alternative would remain unchanged. Rather, different action alternatives would be selected because the goal would refer to more temporally removed events. The modular nature of recent forward architectures (Haruno, Wolpert, \& Kawato, 2001) seems to suggest this second alternative is more likely.

Given these two accounts of temporal extension, what do they imply about integrating information about another's actions with information about one's own? If one assumes that temporal expansion takes place in the predicted consequences of each action alternative, one must also assume that such predictions can be derived for action alternatives that are not currently at one's own disposal, to explain the group case. This implies that one would use one's own action system to simulate the outcomes of other's actions. For the present task this would mean that one uses one's own experience in the braking situation to simulate the other's braking performance. Several studies support this idea (Blakemore \& Decety, 2001; Gallese \& Goldman, 1998; Jeannerod, 1999; Knoblich, Seigerschmidt, Flach, \& Prinz, 2002).

If one assumes that temporal expansion takes place in the goal, simulation of the other's action would take another form. It would be sufficient to generate predictions that take into account the different types of events perceived in the environment, without changing the existing forward models. These predictions could be based on contingencies between one's own actions, other's actions, and the jointly controlled event. As the temporal horizon of these integrated predictions increased, the reference point for forward models simulating the consequences of one's own actions would change. The actions of others would become integrated with one's own on a level of distal events (Jordan \& Knoblich, in press; Knoblich \& Flach, 2001; Knoblich \& Jordan, 2002; Knoblich \& Prinz, 2001).

One advantage of the event integration hypothesis is that it provides a parsimonious assumption about how self- and othergenerated actions can be integrated to achieve a jointly intended outcome. Recent accounts (Hommel et al., 2001; Jordan, 1998, 1999; Jordan, Stork, Knuf, Kerzel, \& Müsseler, 2002; Prinz, 1997) suggest that event codes as postulated by this explanation might play a central role in individual action control. The results of the present experiments indicate that event codes might also play an important role in joint action planning (see also Sebanz, Knoblich, \& Prinz, 2003). It is important to note that they might provide a level at which predictions about one's own and other's actions can be flexibly integrated and temporally extended. In addition, such predictions could be used as a reference or control parameter for forward or inverse models.

\section{Generalizability}

A further point to discuss is the degree to which our tracking task is similar to control demands in real-world situations. Regardless of the task's simplicity we are confident it effectively captures two aspects that characterize a wide variety of real-world situations. First, there is the need to acquire anticipatory control. Successful implementation of an ACS requires that future events become more important to action control than current events. Such a need characterizes many situations that individuals and groups encounter (Dörner, 1990; Reason, 1990). Under many circumstances such predictions become critical for performance. For instance, it is impossible to steer a canoe without taking the other's paddle strokes into account. Otherwise one has a good chance of going around in circles, as novices often do. A second characteristic that our tracking task shares with many real-world situations is the need to perform actions in real time (Heuer, 1996). Without adjusting the timing of one's own actions with regard to the other's actions and the common goal, one cannot steer the canoe.

One difference between our tracking task and many real-world situations is that only a small amount of information was shared between group members. To be sure, the more information one has about the other's action the easier it is to acquire anticipatory control in a group. Regardless of the amount of information available, however, there remains the task of integrating information about events that one can produce and events that others can produce to make jointly intended events happen. Thus, as technologies continue to increase the types of tasks that remotely located individuals can jointly control, as playing an interactive game on the Internet or participating in joint surgery via remote, maximal development of an ACS will probably require access to both the jointly controlled event as well as information regarding the state of the action alternatives owned by the other.

In conclusion, the present results address questions that tend to be overlooked in theories of action control. By focusing on dynamic control in both individual and group cases, we have addressed the expansion of the temporal horizon in action planning - a phenomenon that is essential to the development of an 
ACS in almost any task. Our method of comparing groups and individuals as they perform the exact same task could be used to investigate a whole variety of existing experimental paradigms devoted to the study of action control in the individual, as for instance, task switching (cf. Allport, 1993; Mayr \& Keele, 2000; Meiran, Chorev, \& Sapir, 2000; Rogers \& Monsell, 1995) or dual-tasks paradigms (Meyer \& Kieras, 1997). Such a comparison might allow one to investigate (a) the extent to which individuals represent action alternatives, goals, or task sets they do not have at their disposal and (b) whether one's own and other's actions and goals are coded in a similar or qualitatively distinct manner.

\section{References}

Allport, A. (1993). Attention and control: Have we been asking the wrong questions? In D. E. Meyer \& S. Kornblum (Eds.), Attention and performance XIV: Synergies in experimental psychology, artificial intelligence, and cognitive neuroscience (pp. 183-218). Cambridge, MA: MIT Press.

Bekkering, H., Wohlschlaeger, A., \& Gattis, M. (2000). Imitation of gestures in children is goal-directed. Quarterly Journal of Experimental Psychology: Human Experimental Psychology, 53(A), 153-164.

Blakemore, S.-J., \& Decety, J. (2001). From the perception of action to the understanding of intention. Nature Reviews Neuroscience, 2, 561-567.

Clark, H. H. (1996). Using language. Cambridge, UK: Cambridge University Press.

Dörner, D. (1990). The logic of failure. In D. E. Broadbent \& J. T. Reason (Eds.), Human factors in hazardous situations (pp. 15-36). New York: Oxford University Press.

Gallese, V., \& Goldman, A. (1998). Mirror neurons and the simulation theory of mind reading. Trends in Cognitive Science, 2, 493-501.

Haruno, M., Wolpert, D. M., \& Kawato, M. (2001). MOSAIC model for sensorimotor learning and control. Neural Computation, 13, 2201-2220.

Heuer, H. (1996). Coordination. In H. Heuer \& S. W. Keele (Eds.): Handbook of perception and action: Vol. 2. Motor skills (pp. 121-180). San Diego, CA: Academic Press.

Hommel, B., Müsseler, J., Aschersleben, G., \& Prinz, W. (2001). The theory of event coding (TEC). Behavioral and Brain Sciences, 24, 849-937.

Hommel, B., \& Prinz, W. (1997). (Eds.). Theoretical issues in stimulusresponse compatibility. Amsterdam: North-Holland.

Jeannerod, M. (1999). The 25th Bartlett Lecture: To act or not to act: Perspectives on the representation of actions. Quarterly Journal of Experimental Psychology, Human Experimental Psychology, 52(A), $1-29$.

Jordan, J. S. (1998). Intentionality, perception, and autocatalytic closure: A potential means of repaying psychology's conceptual debt. In J. S. Jordan (Ed.), Systems theories and a priori aspects of perception (pp. 181-208). Amsterdam: Elsevier.

Jordan, J. S. (1999). Cognition and spatial perception: Production of output or control of input? In G. Aschersleben, J. Müsseler, \& T. Bachmann (Eds.), Cognitive contributions to the perception of spatial and temporal events (pp. 69-90). Amsterdam: Elsevier.

Jordan, J. S., \& Knoblich, G. (in press). Spatial perception and control. Psychonomic Bulletin \& Review.
Jordan, J. S., Stork, S., Knuf, L., Kerzel, D., \& Müsseler, J. (2002). Action planning affects spatial localization. In W. Prinz \& B. Hommel (Eds.), Attention and Performance XIX: Common Mechanisms in Perception and Action (pp. 158-176). Oxford, UK: Oxford University Press.

Kelso, J. A. S. (1997). Dynamic patterns: The self-organization of brain and behavior. Cambridge, MA: MIT Press.

Knoblich, G., \& Flach, R. (2001). Predicting the effects of actions: Interactions of perception and action. Psychological Science, 12, 467-472.

Knoblich, G., \& Jordan, J. S. (2002). The mirror system and joint action. In M. I. Stamenov \& V. Gallese (Eds.), Mirror neurons and the evolution of brain and language (pp. 115-124). Amsterdam: John Benjamins.

Knoblich, G., \& Prinz, W. (2001). Recognition of self-generated actions from kinematic displays of drawing. Journal of Experimental Psychology: Human Perception and Performance, 27, 456-465.

Knoblich, G., Seigerschmidt, E., Flach, R., \& Prinz, W. (2002). Authorship effects in the prediction of handwriting strokes: Evidence for action simulation during action perception. Quarterly Journal of Experimental Psychology: Human Experimental Psychology, 55(A), 1027-1046.

Mayr, U., \& Keele, S. W. (2000) Changing internal constraints on action: The role of backward inhibition. Journal of Experimental Psychology: General, 129, 4-26.

Mechsner, F., Kerzel, D., Knoblich, G., \& Prinz, W. (2001, November). What is coordinated in bimanual coordination? Nature, 414, 69-72.

Meiran, N., Chorev, Z., \& Sapir, A. (2000). Component processes in task switching. Cognitive Psychology, 41, 211-253.

Meyer, D. E., \& Kieras, D. E. (1997). A computational theory of executive cognitive processes and multiple-task performance. Psychological Review, 104, 3-65.

Prinz, W. (1997). Perception and action planning. European Journal of Cognitive Psychology, 9, 129-154.

Reason, J. (1990). Human error. New York: Cambridge University Press.

Rogers, R. D., \& Monsell, S. (1995). Costs of a predictable switch between simple cognitive tasks. Journal of Experimental Psychology: General, 124, 207-231.

Rosenbaum, D. A. (1980). Human movement initiation: Specification of arm, direction, and extent. Journal of Experimental Psychology: General, 109, 444-474.

Schmidt, R. A., \& Lee, T. D. (1999). Motor control and learning. Champaign, IL: Human Kinetics.

Schmidt, R. C., Carello, C., \& Turvey, M. T. (1990). Phase transitions and critical fluctuations in the visual coordination of rhythmic movements between people. Journal of Experimental Psychology: Human Perception and Performance, 16, 227-247.

Sebanz, N., Knoblich, G., \& Prinz, W. (2003) Representing others' actions: Just like one's own? Cognition, 88, B11-B21.

Steiner, I. D. (1972). Group process and productivity. New York: Academic Press.

Wolpert, D. M., \& Ghahramani, Z. (2000). Computational principles of movement neuroscience. Nature Neuroscience, 3, 1212-1217.

Wolpert, D. M., \& Kawato, M. (1998). Multiple paired forward and inverse models for motor control. Neural Networks, 11, 1317-1329.

Received July 29, 2002

Revision received January 31, 2003

Accepted March 16, 2003 